MATHEMATICS OF COMPUTATION

Volume 66, Number 218, April 1997, Pages 699-717

S $0025-5718(97) 00828-4$

\title{
THE RESOLUTION OF THE GIBBS PHENOMENON FOR SPHERICAL HARMONICS
}

\author{
ANNE GELB
}

\begin{abstract}
Spherical harmonics have been important tools for solving geophysical and astrophysical problems. Methods have been developed to effectively implement spherical harmonic expansion approximations. However, the Gibbs phenomenon was already observed by Weyl for spherical harmonic expansion approximations to functions with discontinuities, causing undesirable oscillations over the entire sphere.

Recently, methods for removing the Gibbs phenomenon for one-dimensional discontinuous functions have been successfully developed by Gottlieb and Shu. They proved that the knowledge of the first $N$ expansion coefficients (either Fourier or Gegenbauer) of a piecewise analytic function $f(x)$ is enough to recover an exponentially convergent approximation to the point values of $f(x)$ in any subinterval in which the function is analytic.

Here we take a similar approach, proving that knowledge of the first $N$ spherical harmonic coefficients yield an exponentially convergent approximation to a spherical piecewise smooth function $f(\theta, \phi)$ in any subinterval $\left[\theta_{1}, \theta_{2}\right]$, $\phi \in[0,2 \pi]$, where the function is analytic. Thus we entirely overcome the Gibbs phenomenon.
\end{abstract}

\section{INTRODUCTION}

Spherical coordinates arise in many problems studied by geophysicists and astrophysicists. Methods of representation of discrete functions on spheres have been discussed in great detail, as there are many analytical and computational considerations. One should refer to [10] and [11] for details. In this paper we consider the spectral spherical harmonic representation. The spherical harmonics are a two-dimensional basis set that automatically remedy the pole problem and offer exponential convergence for analytic functions on the sphere. Also many numerical algorithms in spherical harmonics already exist. Thus it seems natural to represent a function in spherical coordinates with spherical harmonics.

The spherical harmonic expansion of a function $f(\theta, \phi)$ defined on a sphere with colatitude coordinate $\theta$ and longitude coordinate $\phi, 0 \leq \theta \leq \pi, 0 \leq \phi \leq 2 \pi$, is defined as

Received by the editor October 26, 1995 and, in revised form, May 1, 1996.

1991 Mathematics Subject Classification. Primary 42A10, 41A10, 41A25.

Key words and phrases. Gibbs phenomenon, Gegenbauer polynomials, spherical harmonics, exponential accuracy.

This work was supported in part by AFOSR grant F49620-95-1-0074, NSF grant DMS 95008 14, and DARPA/ONR AASERT grant N00014-93-1-0985.

(C) 1997 American Mathematical Society 
Definition 1.1.

$$
f(\theta, \phi)=\sum_{q=0}^{\infty} \sum_{|\nu| \leq q} a_{q}^{\nu} Y_{q}^{\nu}(\theta, \phi)
$$

where the spherical harmonic $Y_{q}^{\nu}(\theta, \phi)$ of degree $q$ and order $\nu$ is

$$
Y_{q}^{\nu}(\theta, \phi)=\sqrt{\frac{(2 q+1)(q-\nu) !}{4 \pi(q+\nu) !}} P_{q}^{\nu}(\cos \theta) e^{i \nu \phi}
$$

in terms of the associate Legendre functions $P_{q}^{\nu}(\cos \theta)$ [3].

The orthonormality of the spherical harmonics $Y_{q}^{\nu}(\theta, \phi)$ over the sphere imply that the coefficients $a_{q}^{\nu}$ are given by

$$
a_{q}^{\nu}=\int_{0}^{2 \pi} \int_{0}^{\pi} f(\theta, \phi)\left[Y_{q}^{\nu}(\theta, \phi)\right]^{*} \sin \theta d \theta d \phi
$$

where $\left[Y_{q}^{\nu}(\theta, \phi)\right]^{*}$ are the complex conjugates of $Y_{q}^{\nu}(\theta, \phi)$.

The truncated spectral representation of $f(\theta, \phi)$ is

$$
g_{N}(\theta, \phi)=\sum_{q=0}^{N} \sum_{|\nu| \leq q} a_{q}^{\nu} Y_{q}^{\nu}(\theta, \phi) .
$$

Lemma 1.1. If $f(\theta, \phi)$ is infinitely differentiable, then $g_{N}(\theta, \phi)$ converges spectrally to $f(\theta, \phi)$.

The proof of this lemma is presented in [10].

Now suppose $f(\theta, \phi)$ is a discontinuous but piecewise smooth function. The truncated series no longer converges exponentially to $f$. In fact, the Gibbs phenomenon occurs at the point of discontinuity, causing oscillations over the entire sphere (Figure 1) was initially observed by Herman Weyl [12].

In this paper it is shown that the first $(N+1)^{2}$ spherical harmonic coefficients contain enough information to reconstruct a spectrally accurate approximation, provided that $f(\theta, \phi)$ is a piecewise analytic function.

Gottlieb and Shu [7] established exponential convergence and eliminated the Gibbs phenomenon for one-dimensional piecewise analytic functions assuming knowledge of the first $N$ Fourier coefficients or the first $N$ Gegenbauer coefficients (in general). First the Gegenbauer coefficients based on the Gegenbauer polynomials $C_{n}^{\lambda}(x)$, which are orthogonal in $[-1,1]$ with the weight function $\left(1-x^{2}\right)^{\lambda-\frac{1}{2}}$, were obtained from the known Fourier or Gegenbauer coefficients for $\lambda \sim N$, and then the Gegenbauer expansion was constructed. The convergence was shown to be exponential in any subinterval for which the function was analytic. This paper follows the same idea, only now in the two-dimensional spherical coordinates and with knowledge of the first $(N+1)^{2}$ spherical harmonic coefficients. The procedure consists of the same two steps as given in [7]:

1. Using the given first $(N+1)^{2}$ spherical harmonic coefficients, $a_{q}^{\nu}$, based on spherical harmonics $Y_{q}^{\nu}(\theta, \phi)$, we recover, with exponential accuracy, the first $m \sim N$ Gegenbauer expansion coefficients, based on a subinterval $\left[\theta_{1}, \theta_{2}\right] \subset[0, \pi], \phi \in[0,2 \pi]$, in which the function is presumably analytic. This can be achieved for any $L_{1}$ function, as long as $\lambda$, the order of the Gegenbauer polynomial, is chosen to be proportional to $N$. The error 
incurred at this stage is called the truncation error, and is investigated in Section 3.

2. We then apply the previously established exponential convergence proof [6] of the Gegenbauer expansion to analytic functions in $\left[\theta_{1}, \theta_{2}\right], \phi \in$ $[0,2 \pi]$, when the parameter $\lambda$ is proportional to the number of terms retained in the expansion. The error at this stage is labeled the regularization error. Details can be found in [6]. The results are quoted in Section 4.

Section 5 contains a numerical example to illustrate our results. In Section 2 we establish some useful properties of the Gegenbauer polynomials and the associated Legendre functions (Section 2 also appears in [7]).

Throughout this paper, $A$ denotes a generic constant or at most a polynomial in the growing parameters, as will be indicated in the text.

\section{Preliminaries}

2.1. Gegenbauer polynomials. In this section we present some properties of the Gegenbauer polynomials and the associated Legendre functions which are necessary to prove the results in Sections 3 and 4. One should see Bateman [2] for details.

Definition 2.1. The Gegenbauer polynomial $C_{n}^{\lambda}(x)$, for $\lambda \geq 0$, is defined by

$$
\left(1-x^{2}\right)^{\lambda-\frac{1}{2}} C_{n}^{\lambda}(x)=G(\lambda, n) \frac{d^{n}}{d x^{n}}\left[\left(1-x^{2}\right)^{n+\lambda-\frac{1}{2}}\right],
$$

where $G(\lambda, n)$ is given by

$$
G(\lambda, n)=\frac{(-1)^{n} \Gamma\left(\lambda+\frac{1}{2}\right) \Gamma(n+2 \lambda)}{2^{n} n ! \Gamma(2 \lambda) \Gamma\left(n+\lambda+\frac{1}{2}\right)} .
$$

Formula (2.1) is also called the Rodrigues' formula [1, page 175].

Under this definition, for $\lambda>0$,

$$
C_{n}^{\lambda}(1)=\frac{\Gamma(n+2 \lambda)}{n ! \Gamma(2 \lambda)}
$$

and

$$
\left|C_{n}^{\lambda}(x)\right| \leq C_{n}^{\lambda}(1), \quad-1 \leq x \leq 1 .
$$

The Gegenbauer polynomials are orthogonal under the weight function $\left(1-x^{2}\right)^{\lambda-\frac{1}{2}}$, thus

$$
\int_{-1}^{1}\left(1-x^{2}\right)^{\lambda-\frac{1}{2}} C_{k}^{\lambda}(x) C_{n}^{\lambda}(x) d x=\delta_{k, n} h_{n}^{\lambda}
$$

where, for $\lambda>0$,

$$
h_{n}^{\lambda}=\pi^{\frac{1}{2}} C_{n}^{\lambda}(1) \frac{\Gamma\left(\lambda+\frac{1}{2}\right)}{\Gamma(\lambda)(n+\lambda)} .
$$

The approximation of the Gegenbauer polynomials for large $n$ and $\lambda$ is dependent upon the well-known Stirling's formula for $\Gamma(x)$ given by

$$
(2 \pi)^{\frac{1}{2}} x^{x+\frac{1}{2}} e^{-x} \leq \Gamma(x+1) \leq(2 \pi)^{\frac{1}{2}} x^{x+\frac{1}{2}} e^{-x} e^{\frac{1}{12 x}}, \quad x \geq 1 .
$$


Lemma 2.1. There exists a constant $A$ independent of $\lambda$ and $n$ such that

$$
A^{-1} \frac{\lambda^{\frac{1}{2}}}{(n+\lambda)} C_{n}^{\lambda}(1) \leq h_{n}^{\lambda} \leq A \frac{\lambda^{\frac{1}{2}}}{(n+\lambda)} C_{n}^{\lambda}(1) .
$$

The proof follows from (2.6) and Stirling's formula (2.7).

The following lemma to be used later is easily obtained from the Rodrigues formula (2.1).

Lemma 2.2. For any $\lambda \geq 1$

$$
\frac{d}{d x}\left[\left(1-x^{2}\right)^{\lambda-\frac{1}{2}} C_{n}^{\lambda}(x)\right]=\frac{G(\lambda, n)}{G(\lambda-1, n+1)}\left(1-x^{2}\right)^{\lambda-\frac{3}{2}} C_{n+1}^{\lambda-1}(x) .
$$

The proof follows from taking the derivative on both sides of the Rodrigues formula (2.1), and then using it again on the right-hand side.

The following formula [1, page 176] will also be needed:

$$
C_{n}^{\lambda}(x)=\frac{1}{2(n+\lambda)}\left(\frac{d}{d x}\left[C_{n+1}^{\lambda}(x)-C_{n-1}^{\lambda}(x)\right]\right),
$$

which is true for all $\lambda \geq 0$.

The associated Legendre functions are defined as

\section{Definition 2.2.}

$$
P_{l}^{m}(x)=\left(1-x^{2}\right)^{\frac{m}{2}} \frac{d^{m} P_{l}(x)}{d x^{m}}, \quad 0 \leq m \leq l .
$$

Employing Rodrigues' formula for $P_{l}(x)$, the corresponding Rodrigues formula for $P_{l}^{m}(x)$ is

$$
P_{l}^{m}(x)=\frac{\left(1-x^{2}\right)^{\frac{m}{2}}}{2^{l} l !} \frac{d^{l+m}}{d x^{l+m}}\left(x^{2}-1\right)^{l}, \quad 0 \leq m \leq l .
$$

The equation (2.11) allows rapid development of many properties of the $P_{l}^{m}$, particularly the recurrence relations [8, page 1005],

for $l$

$$
(l-m+1) P_{l+1}^{m}-(2 l+1) x P_{l}^{m}+(l+m) P_{l-1}^{m}=0,
$$

and for $m$

$$
\sqrt{1-x^{2}} P_{l}^{m+1}+2 m x P_{l}^{m}+(l+m)(l-m+1) \sqrt{1-x^{2}} P_{l}^{m-1}=0 .
$$

These equations lead to other useful relations as well, notably those used in the truncation error proofs in Section 3.

\section{Truncation ERror in a SUbinterval}

Assume that $f(\theta, \phi)$ is an $L_{1}$ function defined for $\theta \in[0, \pi]$ and $\phi \in[0,2 \pi]$, and that $f(\theta, \phi)$ is periodic in $\phi$ and analytic for $\theta$ in a subinterval $\left[\theta_{1}, \theta_{2}\right] \subset[0, \pi]$.

Also assume that the spherical harmonic partial sum of $f(\theta, \phi)$ is known

$$
g_{N}^{\nu}(\theta, \phi)=\sum_{q=0}^{N} \sum_{|\nu| \leq q} a_{q}^{\nu} Y_{q}^{\nu}(\theta, \phi) .
$$

The spherical harmonics $Y_{q}^{\nu}(\theta, \phi)$ are defined by

$$
Y_{q}^{\nu}(\theta, \phi)=\sqrt{\frac{(2 q+1)(q-\nu) !}{4 \pi(q+\nu) !}} P_{q}^{\nu}(\cos \theta) e^{i \nu \phi},
$$


where $P_{q}^{\nu}(\cos \theta)$ are the associated Legendre functions (2.11).

The coefficients $a_{q}^{\nu}$ in (3.1) are given by

$$
a_{q}^{\nu}=\int_{0}^{2 \pi} \int_{0}^{\pi} f(\theta, \phi)\left[Y_{q}^{\nu}(\theta, \phi)\right]^{*} \sin \theta d \theta d \phi
$$

where $\left[Y_{q}^{\nu}(\theta, \phi)\right]^{*}$ are the complex conjugates of $Y_{q}^{\nu}(\theta, \phi)$.

It has been shown that $g_{N}^{\nu}(\theta, \phi)$ will not converge fast to $f(\theta, \phi)$ if there exist any discontinuities [12].

The coefficients of $f(\theta, \phi)$ must satisfy

Assumption 3.1. $\left|a_{q}^{\nu}\right| \leq A$ independent of $q$.

Note that this is true for $f(\theta, \phi) \in L_{1}$.

The goal is to recover $f(\theta, \phi)$ for $\theta$ in subinterval $\left[\theta_{1}, \theta_{2}\right] \subset[0, \pi]$, and $\phi \in[0,2 \pi]$ for a fixed $\phi$. The approximation expansion is written in terms of $\cos \theta$ because of the associated Legendre functions $P_{q}^{\nu}(\cos \theta)$. Since $\phi$ is fixed, the function $f$ will be considered only as a function of $\theta$ and will be denoted by $f(\theta)$. Hence we make the transformation from $\theta$ in $[0, \pi]$ to $\xi$ in $[-1,1]$ and apply the following definitions.

Definition 3.1. The local variable $\xi=\cos \theta$ is defined by

$$
\begin{aligned}
x(\xi) & =\epsilon \xi+\delta, \\
\epsilon & =\frac{\beta-\alpha}{2}, \\
\delta & =\frac{\beta+\alpha}{2}, \\
-1 & \leq \alpha \leq \beta \leq 1,
\end{aligned}
$$

where $\alpha=\cos \theta_{1}, \beta=\cos \theta_{2}$, and $\theta_{2}<\theta_{1}$.

Definition 3.2. The Gegenbauer partial sum is defined by

$$
f_{m}^{\lambda}(x)=\sum_{l=0}^{m} \hat{f}^{\lambda}(l) C_{l}^{\lambda}(x)
$$

where the first $m$ coefficients $\hat{f}^{\lambda}(l)$ based upon the Gegenbauer polynomials $C_{l}^{\lambda}(x)$ with weight function $\left(1-x^{2}\right)^{\lambda-\frac{1}{2}}$ for any constant $\lambda \geq 0$ are defined by

$$
\hat{f}^{\lambda}(l)=\frac{1}{h_{l}^{\lambda}} \int_{-1}^{1}\left(1-x^{2}\right)^{\lambda-\frac{1}{2}} C_{l}^{\lambda}(x) f(x) d x, \quad 0 \leq l \leq m,
$$

and the Gegenbauer polynomials $C_{l}^{\lambda}(x)$ are defined by (2.1).

The Gegenbauer expansion of $f(x)$ for the subinterval $[\alpha, \beta]$ is

$$
f(\epsilon \xi+\delta)=\sum_{l=0}^{\infty} \hat{f}_{\epsilon}^{\lambda}(l) C_{l}^{\lambda}(\xi), \quad-1 \leq \xi \leq 1,
$$

where the coefficients $\hat{f}_{\epsilon}^{\lambda}(l)$ are

$$
\hat{f}_{\epsilon}^{\lambda}(l)=\frac{1}{h_{l}^{\lambda}} \int_{-1}^{1}\left(1-\xi^{2}\right)^{\lambda-\frac{1}{2}} C_{l}^{\lambda}(\xi) f(\epsilon \xi+\delta) d \xi .
$$


Of course, $\hat{f}_{\epsilon}^{\lambda}(l)$ is not known, but rather an approximation to $\hat{f}_{\epsilon}^{\lambda}(l)$, denoted $\hat{g}_{\epsilon}^{\lambda}(l)$, based on the transformation in equation (3.4) of the spherical harmonic partial sum, $g_{N}^{\nu}(\epsilon \xi+\delta)$. These approximate coefficients $\hat{g}_{\epsilon}^{\lambda}(l)$ are defined as

$$
\hat{g}_{\epsilon}^{\lambda}(l)=\frac{1}{h_{l}^{\lambda}} \int_{-1}^{1}\left(1-\xi^{2}\right)^{\lambda-\frac{1}{2}} C_{l}^{\lambda}(\xi) g_{N}^{\nu}(\epsilon \xi+\delta) d \xi .
$$

The truncation error describes how well the coefficients $\hat{g}_{\epsilon}^{\lambda}(l)$ approximate $\hat{f}_{\epsilon}^{\lambda}(l)$.

Definition 3.3. The truncation error is defined by

$$
T E(\lambda, m, N, \epsilon, \nu)=\max _{-1 \leq \xi \leq 1}\left|\sum_{l=0}^{m}\left(\hat{f}_{\epsilon}^{\lambda}(l)-\hat{g}_{\epsilon}^{\lambda}(l)\right) C_{l}^{\lambda}(\xi)\right|,
$$

where $\hat{f}_{\epsilon}^{\lambda}(l)$ and $\hat{g}_{\epsilon}^{\lambda}(l)$ are defined in equations (3.8) and (3.9).

Lemma 3.1. The truncation error can be estimated by

$$
\begin{aligned}
T E(\lambda, m, N, \epsilon, \nu) \leq \sum_{q=N+1}^{\infty}\left|\sum_{l=0}^{m} \frac{C_{l}^{\lambda}(1)}{h_{l}^{\lambda}} \sum_{|\nu| \leq N}\right| a_{q}^{\nu} \mid \sqrt{\frac{(2 q+1)(q-\nu) !}{4 \pi(q+\nu) !}} \\
\times \int_{-1}^{1}\left(1-\xi^{2}\right)^{\lambda-\frac{1}{2}} C_{l}^{\lambda}(\xi) P_{q}^{\nu}(\epsilon \xi+\delta) d \xi \mid .
\end{aligned}
$$

Proof. Substituting equation (3.8) and equation (3.9) into equation (3.10) yields

$$
\hat{f}_{\epsilon}^{\lambda}(l)-\hat{g}_{\epsilon}^{\lambda}(l)=\frac{1}{h_{l}^{\lambda}} \int_{-1}^{1}\left(1-\xi^{2}\right)^{\lambda-\frac{1}{2}} C_{l}^{\lambda}(\xi)\left(f(\epsilon \xi+\delta)-g_{N}^{\nu}(\epsilon \xi+\delta)\right) d \xi .
$$

Applying the estimate (2.4) on $C_{l}^{\lambda}(\xi)$, and the equations (3.1) and (3.2), the estimate (3.11) is obtained.

It must be shown now that the estimate (3.11) is small. For simplicity of notations denote

$$
S_{q, \nu}^{\lambda, l}=\int_{-1}^{1}\left(1-\xi^{2}\right)^{\lambda-\frac{1}{2}} C_{l}^{\lambda}(\xi) P_{q}^{\nu}(\epsilon \xi+\delta) d \xi
$$

and

$$
I_{q, \nu}^{\lambda, l}=\frac{S_{q, \nu}^{\lambda, l}}{G(\lambda, l)}
$$

where $G(\lambda, l)$ is defined in (2.2).

$S_{q, \nu}^{\lambda, l}$ must be bounded effectively so that the truncation error is small. This is done by finding a recurrence relation for $I_{q, \nu}^{\lambda, l} \sim S_{q, \nu}^{\lambda, l}$, and then an upper bound for $|\nu| \leq N$.

Lemma 3.2. $I_{q, \nu}^{\lambda, l}$ satisfies the following recurrence relation

$$
\begin{aligned}
I_{q, \nu}^{\lambda, l}= & \frac{2}{\epsilon(2 q+1)} \frac{(\nu+1)}{(\nu+2)}\left[\frac{(q+\nu)}{(q-\nu)} I_{q-1, \nu}^{\lambda-1, l+1}-\frac{(q-\nu+1)}{(q+\nu+1)} I_{q+1, \nu}^{\lambda-1, l+1}\right] \\
& +\frac{\nu}{(q-\nu)(q+\nu+1)(\nu+2)} I_{q, \nu+2}^{\lambda, l} .
\end{aligned}
$$

See proof of Lemma 3.2 in the appendix. 
It is now possible to bound $S_{q, \nu}^{\lambda, l}$ of (3.13) by applying Lemma 3.2.

Lemma 3.3. For $S_{q, \nu}^{\lambda, l}$ in (3.13) where $0 \leq \nu \leq N$,

$$
\left|S_{q, \nu}^{\lambda, l}\right| \leq \max \left(\nu^{\rho}, 1\right) \sqrt{\frac{(q+\nu) !}{(q-\nu+1) !}} \frac{(q-l)^{(\lambda-\kappa)}}{\epsilon^{\lambda}} \frac{\Gamma(q-\lambda)}{\Gamma(q)} \frac{|G(\lambda, l)|}{|G(0, l+\lambda)|},
$$

where $G(\lambda, l)$ is defined in (2.2) and subject to the conditions

$$
\begin{array}{r}
4 \leq \lambda \leq q \\
0 \leq l \leq m \leq N \\
0 \leq \nu \leq N<q, \\
\lambda-1>\kappa>2 \\
\rho>3 .
\end{array}
$$

See proof of Lemma 3.3 in the appendix.

Note that the upper bound on $\kappa$ and the lower bound on $\rho$ are required to estimate $S_{q, \nu}^{\lambda, l}$ (see proof of Lemma 3.3 and Lemma 3.4). The lower bound on $\kappa$ ensures convergence of the truncation error (Theorem 3.1). These parameters have not been optimized.

An estimate is also needed for $S_{q, \nu}^{\lambda, l}$, where $-N \leq \nu \leq 0$.

Lemma 3.4. For $S_{q, \nu}^{\lambda, l}$ in (3.13), where $-N \leq \nu \leq 0$,

$$
\left|S_{q, \nu}^{\lambda, l}\right| \leq \max \left(|\nu|^{\rho}, 1\right) \sqrt{\frac{(q+\nu-1) !}{(q-\nu) !}} \frac{(q-l)^{(\lambda-\kappa)}}{\epsilon^{\lambda}} \frac{\Gamma(q-\lambda)}{\Gamma(q)} \frac{|G(\lambda, l)|}{|G(0, l+\lambda)|},
$$

where $G(\lambda, l)$ is defined in (2.2) and subject to the conditions

$$
\begin{array}{r}
4 \leq \lambda \leq q, \\
0 \leq l \leq m \leq N, \\
0 \geq \nu \geq-N, \\
\lambda-1>\kappa>2, \\
\rho>3, \\
|N|<q .
\end{array}
$$

See proof of Lemma 3.4 in the appendix.

Combining Lemma 3.3 and Lemma 3.4 yields the following estimate for $S_{q,|\nu|}^{\lambda, l}$.

Lemma 3.5. For $S_{q,|\nu|}^{\lambda, l}$ in (3.13) we have the following estimate

$$
\left|S_{q,|\nu|}^{\lambda, l}\right| \leq \max \left(|\nu|^{\rho}, 1\right) \sqrt{\frac{(q+\nu) !}{(q-\nu) !}} \frac{(q-l)^{(\lambda-\kappa)}}{\epsilon^{\lambda}} \frac{\Gamma(q-\lambda)}{\Gamma(q)} \frac{|G(\lambda, l)|}{|G(0, l+\lambda)|},
$$


where $G(\lambda, l)$ is defined in (2.2) and subject to the conditions

$$
\begin{array}{r}
4 \leq \lambda \leq q, \\
0 \leq l \leq m \leq N, \\
|\nu| \leq N<q, \\
\lambda-1>\kappa>2, \\
\rho>3 .
\end{array}
$$

Finally it is possible to estimate $\left|S_{q,|\nu|}^{\lambda, l}\right|$ in terms of the truncation error parameters $\lambda, m, \epsilon$, and $\nu$.

\section{Lemma 3.6.}

$$
\left|S_{q,|\nu|}^{\lambda, l}\right| \leq A \frac{(m+2 \lambda)^{m+2 \lambda}}{(2 \epsilon \lambda)^{\lambda} m^{m}} \frac{1}{q^{\kappa}} \sqrt{\frac{(q+\nu) !}{(q-\nu) !}},
$$

where A grows at most as $(m+\lambda)^{\frac{1}{2}} N^{\rho}$. The estimate is subject to the conditions

$$
\begin{array}{r}
4 \leq \lambda \leq q, \\
0 \leq l \leq m \leq N, \\
|\nu| \leq N<q, \\
\lambda-1>\kappa>2, \\
\rho>3 .
\end{array}
$$

Proof. Starting from estimate (3.18) and using the definition of $G(\lambda, l)$ in (2.2), the following estimate is obtained:

$$
\begin{aligned}
\left|S_{q,|\nu|}^{\lambda, l}\right| & \leq \frac{\Gamma(q-\lambda)}{\epsilon^{\lambda} \Gamma(q)} \frac{|G(\lambda, l)|}{|G(0, l+\lambda)|} \sqrt{\frac{(q+\nu) !}{(q-\nu) !}}|\nu|^{\rho}(q-l)^{\lambda-\kappa} \\
\leq & \frac{\Gamma(q-\lambda)}{\epsilon^{\lambda} \Gamma(q)} \frac{\Gamma\left(\lambda+\frac{1}{2}\right) \Gamma(l+2 \lambda)}{2^{l} l ! \Gamma(2 \lambda) \Gamma\left(l+\lambda+\frac{1}{2}\right)} \frac{2^{l+\lambda}(l+\lambda) \Gamma\left(l+\lambda+\frac{1}{2}\right)}{\Gamma(l+\lambda)} \\
& \times \sqrt{\frac{(q+\nu) !}{(q-\nu) !}}|\nu|^{\rho}(q-l)^{\lambda-\kappa} \\
\leq & \frac{\Gamma(q-\lambda)}{\epsilon^{\lambda} \Gamma(q)} \frac{\Gamma\left(\lambda+\frac{1}{2}\right) \Gamma(l+2 \lambda)}{2^{l} l ! \Gamma(2 \lambda) \Gamma\left(l+\lambda+\frac{1}{2}\right)} \frac{2^{l+\lambda}(l+\lambda) \Gamma\left(l+\lambda+\frac{1}{2}\right)}{\Gamma(l+\lambda)} \\
\leq & \frac{\Gamma(q-\lambda)}{\epsilon^{\lambda} \Gamma(q)} \frac{\Gamma(\lambda) \Gamma(l+2 \lambda) 2^{\lambda}}{l ! \Gamma(2 \lambda)} \sqrt{\frac{(q+\nu) !}{(q-\nu) !}}|\nu|^{\rho}(q-l)^{\lambda-\kappa}(q-l)^{\lambda-\kappa} \\
\leq & \frac{\Gamma(q-\lambda)}{\epsilon^{\lambda} \Gamma(q)} \frac{\Gamma(\lambda) \Gamma(m+2 \lambda) 2^{\lambda}}{m ! \Gamma(2 \lambda)} \sqrt{\frac{(q+\nu) !}{(q-\nu) !}}|\nu|^{\rho}(q)^{\lambda-\kappa} \\
\leq & A \frac{(q-\lambda)^{q-\lambda} e^{-(q-\lambda)}}{\epsilon^{\lambda} q^{q} e^{-q}} \frac{\lambda^{\lambda} e^{-\lambda}(m+2 \lambda) m+2 \lambda}{m^{m} e^{-(m+2 \lambda)} 2^{\lambda}} \sqrt{\frac{(q+\nu) !}{(q-\nu) !}} \frac{1}{q^{\kappa}} \\
\leq & A \frac{(m+2 \lambda)^{2 \lambda} e^{-2 \lambda}}{(2 \epsilon \lambda)^{\lambda} m^{m}} \sqrt{\frac{(q+\nu) !}{(q-\nu) !} \frac{1}{q^{\kappa}} .}
\end{aligned}
$$


Equation (2.2) is used in the second inequality, the monotonicity with respect to $l$ in the fourth inequality, and Stirling's formula (2.7) in the fifth inequality.

We are now ready for the main theorem of this section.

Theorem 3.1. Let the truncation error be defined in (3.10). Let $\lambda=\alpha \epsilon N$ and $m=\beta \epsilon N$ with $0<\alpha, \beta<1, \lambda-1>\kappa \geq 2$, and $\rho>3$. Then for $|\nu| \leq N$

$$
T E(\alpha \epsilon N, \beta \epsilon N, N, \epsilon) \leq A\left(\frac{(\beta+2 \alpha)^{\beta+2 \alpha}}{2^{\alpha} \alpha^{\alpha} \beta^{\beta}}\right)^{\epsilon N},
$$

where $A$ grows at most as $N^{1+\rho}$. In particular, if $\alpha=\beta<\frac{2}{27}$, then

$$
T E(\alpha \epsilon N, \alpha \epsilon N, N, \epsilon) \leq A p^{\epsilon N},
$$

where

$$
p=\left(\frac{27 \alpha}{2}\right)^{\alpha}<1
$$

Proof. The theorem follows from the definition of the truncation error (3.11)

$$
\begin{aligned}
T E(\lambda, m, N, \epsilon, \nu) \leq \sum_{q=N+1}^{\infty} \mid & \sum_{l=0}^{m} \frac{C_{l}^{\lambda}(1)}{h_{l}^{\lambda}} \sum_{|\nu| \leq N}\left|a_{q}^{\nu}\right| \sqrt{\frac{(2 q+1)(q-\nu) !}{4 \pi(q+\nu) !}} \\
& \times \int_{-1}^{1}\left(1-\xi^{2}\right)^{\lambda-\frac{1}{2}} C_{l}^{\lambda}(\xi) P_{q}^{\nu}(\epsilon \xi+\delta) d \xi \mid .
\end{aligned}
$$

Recalling the bound on $S_{q, \nu}^{\lambda, l}$ in $(3.19)$

$$
\left|S_{q,|\nu|}^{\lambda, l}\right| \leq A \frac{(m+2 \lambda)^{m+2 \lambda}}{(2 \epsilon \lambda)^{\lambda} m^{m}} \frac{1}{q^{\kappa}} \sqrt{\frac{(q+\nu) !}{(q-\nu) !}}
$$

the assumption (3.1)

$$
\left|a_{q}^{\nu}\right| \leq A
$$

and the estimate of $h_{n}^{\lambda}$ in (2.8)

$$
A^{-1} \frac{\lambda^{\frac{1}{2}}}{(n+\lambda)} C_{n}^{\lambda}(1) \leq h_{n}^{\lambda} \leq A \frac{\lambda^{\frac{1}{2}}}{(n+\lambda)} C_{n}^{\lambda}(1),
$$

the truncation error estimate is obtained

$$
T E(\lambda, m, N, \epsilon, \nu) \leq A \sum_{q=N+1}^{\infty} \sum_{|\nu| \leq N} \sqrt{\frac{(2 q+1)}{4 \pi}} \frac{(m+2 \lambda)^{m+2 \lambda}}{(2 \epsilon \lambda)^{\lambda} m^{m}} \frac{1}{q^{\kappa}} .
$$

Substituting in the values $\lambda=\alpha \epsilon N$ and $m=\beta \epsilon N$,

$$
T E(\alpha \epsilon N, \beta \epsilon N, N, \epsilon) \leq A\left(\frac{(\beta+2 \alpha)^{\beta+2 \alpha}}{2^{\alpha} \alpha^{\alpha} \beta^{\beta}}\right)^{\epsilon N},
$$

where $A$ grows at most as $N^{1+\rho}$. 


\section{Regularization ERROR}

For the one-dimensional case, as shown in [7], the second part of the error, called the regularization error, is caused by the finite Gegenbauer expansion based on a subinterval $[\alpha, \beta] \subset[-1,1]$ approximation to a function $f(x)$ which is assumed analytic in this sub-interval. Instead of $f(x)$ on a subinterval $[\alpha, \beta] \subset[-1,1]$ as in [7], we now have $f(\theta, \phi)$ on a subinterval $\left[\theta_{1}, \theta_{2}\right] \subset[0, \pi], \phi \in[0,2 \pi]$. The regularization error will be the same result as shown in [7]. We will thus just quote the result (substituting $f(\theta, \phi)$ for $f(x)$ ).

Assume that $f(\theta, \phi)$ is an analytic function on $\left[\theta_{1}, \theta_{2}\right] \subset[0, \pi], \phi \in[0,2 \pi]$, satisfying:

Assumption 4.1. There exist constants $\tau \geq 1$ and $C(\tau)$ such that, for every $k \geq 0$,

$$
\max _{\theta_{1} \leq \theta \leq \theta_{2}}\left|\frac{d^{k} f}{d x^{k}}(\theta, \phi)\right| \leq C(\tau) \frac{k !}{\tau^{k}}, \quad 0 \leq \phi \leq 2 \pi .
$$

This is a standard assumption for analytic functions. $\tau$ is the distance from $\left[\theta_{1}, \theta_{2}\right]$ to the nearest singularity of $f(\theta, \phi)$ in the complex plane (see for example $[9])$.

As in the case of the truncation error, the approximation expansion is in terms of $\cos \theta$. Recall the transformation that was made previously from $\theta$ in $[0, \pi]$ to the local variable $\xi$ in $[-1,1]$ :

$$
\begin{aligned}
x(\xi) & =\epsilon \xi+\delta, \\
\epsilon & =\frac{\beta-\alpha}{2}, \\
\delta & =\frac{\beta+\alpha}{2} m, \\
-1 & \leq \alpha \leq \beta \leq 1,
\end{aligned}
$$

where $\alpha=\cos \theta_{1}, \beta=\cos \theta_{2}$, and $\theta_{2}<\theta_{1}$.

Consider the Gegenbauer partial sum of the first $m$ terms for the function $f(\epsilon \xi+\delta)$ :

$$
f_{m}^{\lambda}(\xi)=\sum_{l=0}^{m} \hat{f}_{\epsilon}^{\lambda}(l) C_{l}^{\lambda}(\xi)
$$

with $\xi, \epsilon$ and $\delta$ defined above, and the Gegenbauer coefficients based on $[\alpha, \beta]$ defined by

$$
\hat{f}_{\epsilon}^{\lambda}(l)=\frac{1}{h_{l}^{\lambda}} \int_{-1}^{1}\left(1-x^{2}\right)^{\lambda-\frac{1}{2}} C_{l}^{\lambda}(\xi) f(\epsilon \xi+\delta) d \xi .
$$

The regularization error in the maximum norm is defined by:

$$
R E(\lambda, m, \epsilon)=\max _{-1 \leq \xi \leq 1}\left|f(\epsilon \xi+\delta)-\sum_{l=0}^{m} \hat{f}_{\epsilon}^{\lambda}(l) C_{l}^{\lambda}(\xi)\right| .
$$

The following estimate of the regularization error, when $\lambda \sim m$, is obtained:

Theorem 4.1. Assume $\lambda=\gamma m$ where $\gamma$ is a positive constant. If $f(\theta, \phi)$ is analytic in $\left[\theta_{1}, \theta_{2}\right] \subset[0, \pi], \phi \in[0,2 \pi]$, and satisfies Assumption 4.1, then the regularization error defined in (4.4) can be bounded by

$$
R E(\gamma m, m, \epsilon) \leq A q^{m},
$$


where $q$ is given by

$$
q=\frac{\epsilon(1+2 \gamma)^{1+2 \gamma}}{\tau 2^{1+2 \gamma} \gamma^{\gamma}(1+\gamma)^{1+\gamma}},
$$

which is always less than 1. In particular, if $\gamma=1$ and $m=\beta \in N$ where $\beta$ is a positive constant, then

$$
R E(\beta N, \beta N, \epsilon) \leq A q^{\epsilon N}
$$

with

$$
q=\left(\frac{27 \epsilon}{32 \tau}\right)^{\beta}
$$

Combining the estimates for truncation error and regularization error it is possible to obtain the main theorem of this chapter, showing the complete removal of the Gibbs phenomenon for a discontinuous function on a sphere.

Theorem 4.2. Consider a $L_{1}$ function $f(\theta, \phi)$ analytic in $\left[\theta_{1}, \theta_{2}\right] \subset[0, \pi], \phi \in$ $[0,2 \pi]$, and satisfying Assumption 4.1. Assume that the first $N$ spherical harmonic coefficients

$$
a_{q}^{\nu}=\int_{0}^{2 \pi} \int_{0}^{\pi} f(\theta, \phi)\left[Y_{q}^{\nu}(\theta, \phi)\right]^{*} \sin \theta d \theta d \phi
$$

for $|\nu| \leq N \leq q$, are known. Let $\hat{g}_{\epsilon}^{\lambda}(l)$, defined in (3.9) for $0 \leq l \leq m$ be the Gegenbauer expansion coefficients of the Gegenbauer partial sum, defined in (3.5), based on the subinterval $\left[\theta_{1}, \theta_{2}\right], \phi \in[0,2 \pi]$. Then for $\lambda=m=\beta \epsilon N$ with $\beta<\frac{2}{27}$,

$$
\max _{-1 \leq \xi \leq 1}\left|f(\epsilon \xi+\delta)-\sum_{l=0}^{m} \hat{g}_{\epsilon}^{\lambda}(l) C_{l}^{\lambda}(\xi)\right| \leq A\left(q_{T}^{\epsilon N}+q_{R}^{\epsilon N}\right),
$$

where

$$
q_{T}=\left(\frac{27 \beta}{2}\right)^{\beta}<1, \quad q_{R}=\left(\frac{27 \epsilon}{32 \tau}\right)^{\beta}<1,
$$

and $A$ grows at most as $N^{1+\rho}$, where $\rho>3$.

Proof. Just combine the results of Theorem 3.1 and Theorem 4.1.

\section{NUMERICAL RESUlts}

In this section we show numerical results for one example. Suppose we are given the function

$$
f(\theta, \phi)= \begin{cases}\cos \frac{3 \theta}{2}+\sin \frac{11 \phi}{4}, & 0 \leq \theta \leq \frac{\pi}{2}, \\ \sin \phi, & \frac{\pi}{2}<\theta \leq \pi .\end{cases}
$$

Note the discontinuity at $\theta=\frac{\pi}{2}$. We use $N=20,40$, and 80 latitudinal points $\theta \subset[0, \pi]$, and 36 longitudinal points $\phi \subset[0,2 \pi]$.

Notice in Figure 1 that the Gibbs phenomenon is prevalent at both boundaries as well as in the middle. The spherical harmonic expansion of $f(\theta, \phi)$ produce the $L_{1}$ errors displayed in Table 1 . Although the errors away from the discontinuity appear to slowly converge, oscillations can be seen all over the sphere. 


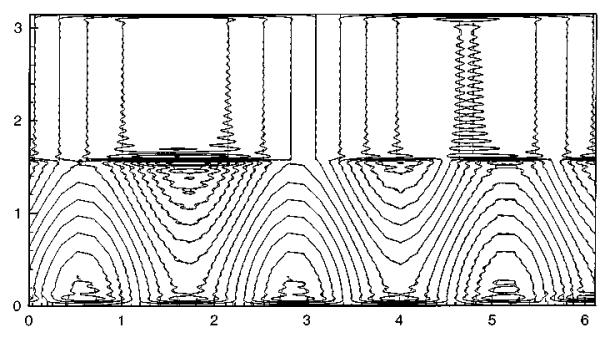

(1a)

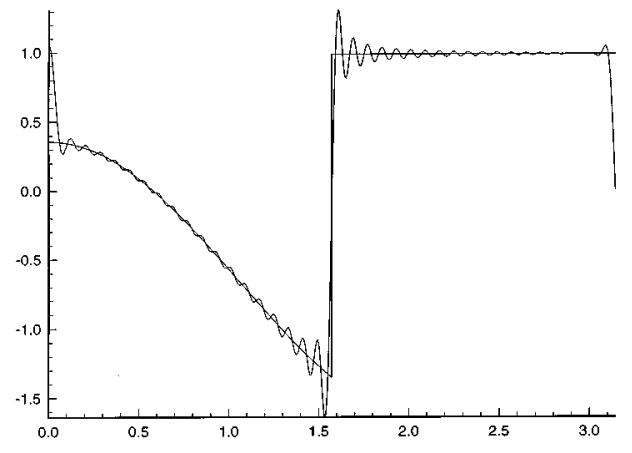

(1b)

FiguRE 1. Spherical harmonic approximation for 80 latitudinal points. (1a) is the contour plot, (1b) is the cut at $\phi=\frac{\pi}{2}$

TABLE 1. $L_{1}$ errors for the spherical harmonic expansion of the function $f(\theta, \phi)$

\begin{tabular}{|l|l|c|c|c|}
\hline$N$ & average error & error at $\frac{\pi}{4}$ & error at $\frac{\pi}{2}$ & error at 0 \\
\hline 20 & .16 & $6.3 \times 10^{-2}$ & .75 & .96 \\
\hline 40 & $9.7 \times 10^{-2}$ & $1.3 \times 10^{-2}$ & .73 & .96 \\
\hline 80 & $6.1 \times 10^{-2}$ & $1.9 \times 10^{-2}$ & .72 & .96 \\
\hline
\end{tabular}

Fixing $\phi=\phi_{f} \in[0,2 \pi]$, the Gegenbauer approximation is obtained in the following way:

1. The spherical harmonic expansion approximation $g_{N}^{\nu}\left(\theta, \phi_{f}\right)$ on the subinterval $\left[\theta_{1}, \theta_{2}\right]$ is computed from the given spherical harmonic coefficients $a_{q}^{\nu}$.

2. The first $m \sim N$ Gegenbauer coefficients for each fixed value $\phi_{f}$

$$
\hat{g}_{\epsilon}^{\lambda}(l)=\frac{1}{h_{l}^{\lambda}} \int_{-1}^{1}\left(1-\xi^{2}\right)^{\lambda-\frac{1}{2}} C_{l}^{\lambda}(\xi) g_{N}^{\nu}\left(\epsilon \xi+\delta, \phi_{f}\right) d \xi
$$

are approximated for $\lambda \sim N$.

3. Finally the Gegenbauer expansion approximation

$$
g_{m}^{\lambda, \epsilon}\left(\theta, \phi_{f}\right)=\sum_{l=0}^{m} \hat{g}_{\epsilon}^{\lambda}(l) C_{l}^{\lambda}(\xi)
$$

is formed for the first $m$ Gegenbauer polynomials.

The results shown in Figure 2 show that the Gegenbauer polynomial harmonic expansion approximation simultaneously eliminates the oscillations at both boundaries (for each subinterval).

The $L_{1}$ errors of the Gegenbauer expansion where $N$ is the number of latitudinal points, $\lambda$ is the order of the Gegenbauer polynomial, and $m$ is the number of Gegenbauer polynomials is seen in Figure 3 and in Table 2. (The number of longitudinal points remains constant.) The errors listed in both of the tables are for the interval $\left[0, \frac{\pi}{2}\right]$. 


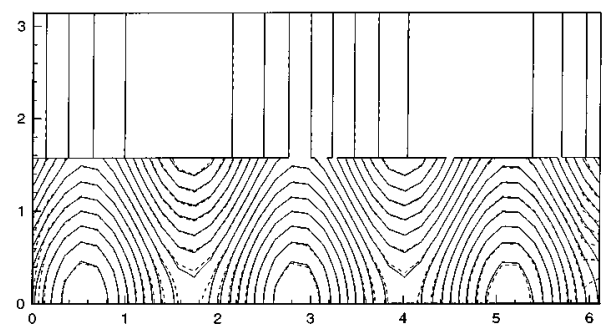

(2a)

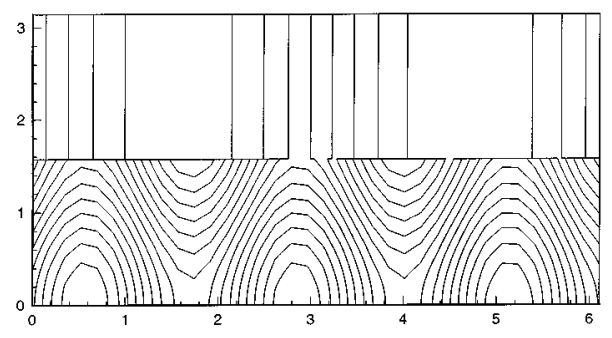

$(2 \mathrm{~b})$

Figure 2. (2a) Gegenbauer approximation for 40 latitudinal points. (2b) Gegenbauer approximation for 80 latitudinal points



Figure 3. Errors in log scale for the Gegenbauer expansion approximation of $f(\theta, \phi)$. The solutions for each subinterval $\left[0, \frac{\pi}{2}\right]$, $\left[\frac{\pi}{2}, \pi\right]$ are found separately for $\phi=\frac{\pi}{2}$

TABLE 2. Errors for the Gegenbauer approximation of the function $f(\theta, \phi)$ using 36 longitudinal points

\begin{tabular}{|c|l|l|c|c|c|c|}
\hline$N$ & $\lambda$ & $m$ & average error & error at $\frac{\pi}{4}$ & error at $\frac{\pi}{2}$ & error at 0 \\
\hline 20 & 1 & 2 & $1.9 \times 10^{-2}$ & $1.1 \times 10^{-3}$ & $8.6 \times 10^{-2}$ & .39 \\
\hline 40 & 7 & 3 & $6.3 \times 10^{-4}$ & $7.4 \times 10^{-6}$ & $3.3 \times 10^{-3}$ & $6.1 \times 10^{-4}$ \\
\hline 80 & 8 & 5 & $5.2 \times 10^{-5}$ & $1.2 \times 10^{-7}$ & $2.4 \times 10^{-4}$ & $3.4 \times 10^{-6}$ \\
\hline
\end{tabular}




\section{Notes}

This paper did not address some important considerations, which will be addressed in future papers:

1. The parameters $\lambda$ and $m$ have not been optimized.

2. The approximation gets worse for large latitudinal $N$. The error seems to occur in the spherical harmonic approximation, where there is a considerable amount of computation taking place. We used the subroutines from the NCAR library to compute the spherical harmonic partial sum.

3 . The errors are an order of magnitude worse at the discontinuities than anywhere else. This is of course to be expected, but there are possible ways to improve upon this error that have not been addressed.

4. We are assuming exact knowledge of the points of discontinuity. When this information is not available, methods for locating discontinuities must be employed.

The author would like to acknowledge David Gottlieb for his enthusiastic support and consistently helpful advice.

\section{APPENDIX}

Lemma 3.2. $I_{q, \nu}^{\lambda, l}$ satisfies the following recurrence relation:

$$
\begin{aligned}
I_{q, \nu}^{\lambda, l}= & \frac{2(\nu+1)}{(\nu+2) \epsilon(2 q+1)}\left[\frac{(q+\nu)}{(q-\nu)} I_{q-1, \nu}^{\lambda-1, l+1}-\frac{(q-\nu+1)}{(q+\nu+1)} I_{q+1, \nu}^{\lambda-1, l+1}\right] \\
& +\frac{\nu}{(q-\nu)(q+\nu+1)(\nu+2)} I_{q, \nu+2}^{\lambda, l}
\end{aligned}
$$

Proof. $P_{q}^{\nu}(t)$, for $t \in[-1,1]$, is defined in [8] by

$$
P_{q}^{\nu}(t)=H(\nu)\left(1-t^{2}\right)^{\frac{\nu}{2}} C_{q-\nu}^{\nu+\frac{1}{2}}(t),
$$

where

$$
H(\nu)=\frac{(-1)^{\nu}(2 \nu) !}{2^{\nu} \nu !} .
$$

Also the following relations for the associated Legendre functions are given in [8]

$$
\frac{\nu t}{\sqrt{1-t^{2}}} P_{q}^{\nu+1}(t)=-\left[\frac{\nu}{2(\nu+1)} P_{q}^{\nu+2}(t)+\frac{\nu(q+\nu+1)(q-\nu)}{2(\nu+1)} P_{q}^{\nu}(t)\right] .
$$

$$
\sqrt{1-t^{2}} P_{q}^{\nu+1}(t)=\frac{1}{2 q+1}\left[(q-\nu)(q-\nu+1) P_{q+1}^{\nu}(t)-(q+\nu+1)(q+\nu) P_{q-1}^{\nu}(t)\right] .
$$

For clarification purposes, let $x=x(\xi)=\epsilon \xi+\delta$, and also

$$
\begin{aligned}
a & =(q-\nu), \\
b & =(q+\nu), \\
c & =(q-\nu+1), \\
d & =(q+\nu+1) .
\end{aligned}
$$


The definition of $P_{q}^{\nu}(t)$ in equation (A.1) is substituted into equation (3.13) to obtain

$$
S_{q, \nu}^{\lambda, l}=H(\nu) \int_{-1}^{1}\left(1-\xi^{2}\right)^{\lambda-\frac{1}{2}} C_{l}^{\lambda}(\xi)\left(1-x^{2}\right)^{\frac{\nu}{2}} C_{q-\nu}^{\nu+\frac{1}{2}}(x) d \xi
$$

Then applying the Rodrigues formula (2.1) and integrating by parts yields

$$
\begin{gathered}
=G\left(\nu+\frac{1}{2}, q-\nu\right) H(\nu) \int_{-1}^{1}\left(1-\xi^{2}\right)^{\lambda-\frac{1}{2}} C_{l}^{\lambda}(\xi)\left(1-x^{2}\right)^{-\frac{\nu}{2}} \frac{d^{q-\nu}}{d x^{q-\nu}}\left(1-x^{2}\right)^{q} d \xi \\
=-\frac{G\left(\nu+\frac{1}{2}, q-\nu\right) H(\nu)}{\epsilon^{q-\nu}} \int_{-1}^{1} \frac{d}{d \xi}\left[\left(1-\xi^{2}\right)^{\lambda-\frac{1}{2}} C_{l}^{\lambda}(\xi)\left(1-x^{2}\right)^{-\frac{\nu}{2}}\right] \\
\times \frac{d^{q-\nu-1}}{d x^{q-\nu-1}}\left(1-x^{2}\right)^{q} d \xi .
\end{gathered}
$$

The equation (2.9) is used and Rodrigues' formula (2.1) is applied again to obtain

$$
\begin{aligned}
= & \frac{H(\nu)(2 \nu+1)}{\epsilon a d} \frac{G(\lambda, l)}{G(\lambda-1, l+1)} \int_{-1}^{1}\left(1-\xi^{2}\right)^{\lambda-\frac{3}{2}} C_{l+1}^{\lambda-1}(\xi) C_{q-\nu-1}^{\nu+\frac{3}{2}}(x)\left(1-x^{2}\right)^{\frac{\nu}{2}+1} \\
& +\frac{H(\nu)(2 \nu+1)}{a d} \int_{-1}^{1} \nu x\left(1-\xi^{2}\right)^{\lambda-\frac{1}{2}} C_{l}^{\lambda}(\xi) C_{q-\nu-1}^{\nu+\frac{3}{2}}(x)\left(1-x^{2}\right)^{\frac{\nu}{2}} d \xi .
\end{aligned}
$$

Applying the definition of $G(\lambda, l)$ in $(2.2)$ and the definition of $P_{q}^{\nu}(t)$ in (A.1) yields

$$
\begin{aligned}
= & -\left(\frac{1}{a c}\right) \frac{G(\lambda, l)}{\epsilon G(\lambda-1, l+1)} \int_{-1}^{1}\left(1-\xi^{2}\right)^{\lambda-\frac{3}{2}} C_{l+1}^{\lambda-1}(\xi) P_{q}^{\nu+1}(x) \sqrt{1-x^{2}} d \xi \\
& +\int_{-1}^{1}\left(1-\xi^{2}\right)^{\lambda-\frac{1}{2}} C_{l}^{\lambda}(\xi) P_{q}^{\nu+1}(x) \frac{\nu x}{\sqrt{1-x^{2}}} d \xi .
\end{aligned}
$$

Using equations (A.3) and (A.4) gives

$$
=\frac{G(\lambda, l)}{\epsilon(2 q+1) G(\lambda-1, l+1)} \int_{-1}^{1}\left(1-\xi^{2}\right)^{\lambda-\frac{3}{2}} C_{l+1}^{\lambda-1}(\xi)\left(\frac{b}{a} P_{q-1}^{\nu}(x)-\frac{c}{d} P_{q+1}^{\nu}(x)\right) d \xi
$$

$$
+\frac{\nu}{2(\nu+1)} \int_{-1}^{1}\left(1-\xi^{2}\right)^{\lambda-\frac{1}{2}} C_{l}^{\lambda}(\xi)\left(\frac{1}{a d} P_{q}^{\nu+2}(x)-P_{q}^{\nu}(x)\right) d \xi
$$

Finally substituting equation (3.13) into equation (A.5) yields

$$
\begin{aligned}
I_{q, \nu}^{\lambda, l}= & \frac{1}{\epsilon(2 q+1)}\left[\frac{(q+\nu)}{(q-\nu)} I_{q-1, \nu}^{\lambda-1, l+1}-\frac{(q-\nu+1)}{(q+\nu+1)} I_{q+1, \nu}^{\lambda-1, l+1}\right] \\
& +\frac{\nu}{2(\nu+1)(q-\nu)(q+\nu+1)} I_{q, \nu+2}^{\lambda, l}+\frac{\nu}{2(\nu+1)} I_{q, \nu}^{\lambda, l} .
\end{aligned}
$$


Lemma 3.3. For $S_{q, \nu}^{\lambda, l}$ in ( 3.13) where $0 \leq \nu \leq N$ we have:

$$
\left|S_{q, \nu}^{\lambda, l}\right| \leq \max \left(\nu^{\rho}, 1\right) \sqrt{\frac{(q+\nu) !}{(q-\nu+1) !}} \frac{(q-l)^{(\lambda-\kappa)}}{\epsilon^{\lambda}} \frac{\Gamma(q-\lambda)}{\Gamma(q)} \cdot \frac{|G(\lambda, l)|}{|G(0, l+\lambda)|}
$$

where $G(\lambda, l)$ is defined in (2.2) and subject to the conditions:

$$
\begin{array}{r}
4 \leq \lambda \leq q, \\
0 \leq l \leq m \leq N, \\
0 \leq \nu \leq N<q, \\
\lambda-1>\kappa>2, \\
\rho>3 .
\end{array}
$$

Proof. (by induction) For $\nu=0$

$$
\begin{aligned}
S_{q, \nu}^{\lambda, l} & =\int_{-1}^{1}\left(1-\xi^{2}\right)^{\lambda-\frac{1}{2}} C_{l}^{\lambda}(\xi) P_{q}(\epsilon \xi+\delta) d \xi \\
& =\int_{-1}^{1}\left(1-\xi^{2}\right)^{\lambda-\frac{1}{2}} C_{l}^{\lambda}(\xi) C_{q}^{\frac{1}{2}}(\epsilon \xi+\delta) d \xi
\end{aligned}
$$

Upon defining

$$
F_{q, \mu}^{\lambda, l}=\int_{-1}^{1}\left(1-\xi^{2}\right)^{\lambda-\frac{1}{2}} C_{l}^{\lambda}(\xi) C_{q}^{\mu}(\epsilon \xi+\delta) d \xi,
$$

the equality holds

$$
S_{q, \nu=0}^{\lambda, l}=F_{q, \frac{1}{2}}^{\lambda, l},
$$

$F_{q, \mu}^{\lambda, l}$ is the integral evaluated in the Legendre case, [7]. Using Lemma 3.7 of [7]

$$
\left|F_{q}^{\lambda, l}\right| \leq A \frac{\Gamma(q-\lambda)}{\epsilon^{\lambda} \Gamma(q)} \frac{|G(\lambda, l)|}{|G(0, l+\lambda)|}
$$

where $A$ grows at most as $q^{2 \mu-1}$ and $G(\lambda, l)$ is defined in (2.2). Therefore for $\nu=0$

$$
\left|S_{q, 0}^{\lambda, l}\right| \leq \sqrt{\frac{q !}{(q+1) !}} \frac{(q-l)^{(\lambda-\kappa)}}{\epsilon^{\lambda}} \frac{\Gamma(q-\lambda)}{\Gamma(q)} \frac{|G(\lambda, l)|}{|G(0, l+\lambda)|} .
$$

Applying the recurrence relation for $I_{q, \nu}^{\lambda, l}$ in (3.15) to $S_{q, \nu}^{\lambda, l}$ yields

$$
\begin{aligned}
S_{q, \nu+2}^{\lambda, l}= & \frac{(\nu+2)(q-\nu)(q+\nu+1)}{\nu} S_{q, \nu}^{\lambda, l} \\
& +\frac{G(\lambda, l)}{G(\lambda-1, l-1)} \frac{2(\nu+1)}{\nu \epsilon(2 q+1)} \\
& \times\left[(q-\nu)(q-\nu+1) S_{q+1, \nu}^{\lambda-1, l+1}-(q+\nu)(q+\nu+1) S_{q-1, \nu}^{\lambda-1, l+1}\right] .
\end{aligned}
$$


Thus

$$
\begin{aligned}
\left|S_{q, \nu+2}^{\lambda, l}\right| \leq & \frac{\nu+2}{\nu}\left(\nu^{\rho}\right)(q-\nu)(q+\nu+1) \sqrt{\frac{(q+\nu) !}{(q-\nu+1) !}} \frac{(q-l)^{\lambda-\kappa}}{\epsilon^{\lambda}} \\
& \times \frac{\Gamma(q-\lambda)}{\Gamma(q)} \frac{|G(\lambda, l)|}{|G(0, l+\lambda)|} \\
& +\frac{1}{\epsilon^{\lambda}} \frac{(\nu+1)\left(\nu^{\rho}\right)}{\nu} \frac{|G(\lambda, l)|}{|G(0, l+\lambda)|} \\
& \times\left[\frac{(q-\nu)(q-\nu+1)}{q} \frac{\Gamma(q+2-\lambda)}{\Gamma(q+1)} \sqrt{\frac{(q+1+\nu) !}{(q+2-\nu) !}}(q-l)^{\lambda-\kappa-1}\right. \\
& \left.+\frac{(q+\nu)(q+\nu+1)}{q} \frac{\Gamma(q-\lambda)}{\Gamma(q-1)} \sqrt{\frac{(q-1+\nu) !}{(q-\nu) !}}(q-l-2)^{\lambda-\kappa-1}\right],
\end{aligned}
$$

and therefore

$$
\begin{aligned}
\left|S_{q, \nu+2}^{\lambda, l}\right| \leq & {\left[(\nu+2)^{\rho} \sqrt{\left.\frac{(q+\nu+2) !}{(q-\nu-1) !} \frac{(q-l)^{(\lambda-\kappa)}}{\epsilon^{\lambda}} \frac{\Gamma(q-\lambda)}{\Gamma(q)} \frac{|G(\lambda, l)|}{|G(0, l+\lambda)|}\right]}\right.} \\
& \times\left[\left(\frac{\nu}{\nu+2}\right)^{\rho-1} \frac{(q-\nu)(q+\nu+1)}{\sqrt{(q+\nu+2)(q+\nu+1)(q-\nu+1)(q-\nu)}}\right. \\
& +\frac{(\nu+1) \nu^{\rho-1}}{(\nu+2)^{\rho-1}} \\
& \times\left(\frac{(q-\nu)(q-\nu+1)(q-\lambda+1)(q-\lambda)}{\sqrt{(q+\nu+2)(q-\nu+2)(q-\nu+1)(q-\nu)}(q-l) q^{2}}\right. \\
& \left.\left.+\frac{(q+\nu)(q+\nu+1)(q-1)}{\sqrt{(q+\nu+2)(q+\nu+1)(q+\nu)(q-\nu)}(q-l-2) q}\right)\right] .
\end{aligned}
$$

Hence

$$
\left|S_{q, \nu+2}^{\lambda, l}\right| \leq(\nu+2)^{\rho} \sqrt{\frac{(q+\nu+2) !}{(q-\nu-1) !}} \frac{(q-l)^{(\lambda-\kappa)}}{\epsilon^{\lambda}} \frac{\Gamma(q-\lambda)}{\Gamma(q)} \frac{|G(\lambda, l)|}{|G(0, l+\lambda)|} .
$$

Lemma 3.4. For $S_{q, \nu}^{\lambda, l}$ in (3.13) where $-N \leq \nu \leq 0$ we have:

$$
\left|S_{q, \nu}^{\lambda, l}\right| \leq \max \left(|\nu|^{\rho}, 1\right) \sqrt{\frac{(q+\nu-1) !}{(q-\nu) !} \frac{(q-l)^{(\lambda-\kappa)}}{\epsilon^{\lambda}} \frac{\Gamma(q-\lambda)}{\Gamma(q)}} \cdot \frac{|G(\lambda, l)|}{|G(0, l+\lambda)|}
$$

where $G(\lambda, l)$ is defined in (2.2) and subject to the conditions:

$$
\begin{array}{r}
4 \leq \lambda \leq q, \\
0 \leq l \leq m \leq N, \\
\lambda-1>\kappa>2, \\
0 \geq \nu \geq-N, \\
\rho>3, \\
|N|<q .
\end{array}
$$


Proof. Starting with the recurrence relation

$$
\begin{aligned}
S_{q, \nu-2}^{\lambda, l}= & \frac{(\nu-2)}{\nu(q-\nu+2)(q+\nu-1)} S_{q, \nu}^{\lambda, l} \\
& +\frac{G(\lambda, l)}{G(\lambda-1, l-1)} \frac{2(\nu-1)}{\nu \epsilon(2 q+1)} \frac{1}{(q-\nu+2)(q+\nu-1)}\left[S_{q+1, \nu}^{\lambda-1, l+1}-S_{q-1, \nu}^{\lambda-1, l+1}\right] .
\end{aligned}
$$

Choosing $\eta=-\nu$, so that $0 \leq \eta \leq N$, the following recurrence relation is obtained

$$
\begin{aligned}
S_{q,-(\eta+2)}^{\lambda, l}= & \frac{(\eta+2)}{\eta(q+\eta+2)(q-\eta-1)} S_{q,-\eta}^{\lambda, l} \\
& +\frac{G(\lambda, l)}{G(\lambda-1, l-1)} \frac{2(\eta+1)}{\eta \epsilon(2 q+1)} \frac{1}{(q+\eta+2)(q-\eta-1)} \\
& \times\left[S_{q+1,-\eta}^{\lambda-1, l+1}-S_{q-1,-\eta}^{\lambda-1, l+1}\right] .
\end{aligned}
$$

It must be shown that

$$
\left|S_{q,-\eta}^{\lambda, l}\right| \leq \max \left(|\eta|^{\rho}, 1\right) \sqrt{\frac{(q-\eta-1) !}{(q+\eta) !}} \frac{(q-l)^{(\lambda-\kappa)}}{\epsilon^{\lambda}} \frac{\Gamma(q-\lambda)}{\Gamma(q)} \frac{|G(\lambda, l)|}{|G(0, l+\lambda)|},
$$

where $G(\lambda, l)$ is defined in (2.2) and with the same parameters

$$
\begin{array}{r}
4 \leq \lambda \leq q, \\
0 \leq l \leq m \leq N, \\
0 \leq \eta \leq N<q, \\
\lambda-1>\kappa>2, \\
\rho>3 .
\end{array}
$$

For $\eta=0$ the same first inductive step as in Lemma 3.3 is used.

Substituting into the recurrence relation (A.7) above, the inequality is directly obtained

$$
\left|S_{q,-(\eta+2)}^{\lambda, l}\right| \leq(\eta+2)^{\rho} \sqrt{\frac{(q-\eta-3) !}{(q+\eta+2) !}} \frac{(q-l)^{(\lambda-\kappa)}}{\epsilon^{\lambda}} \frac{\Gamma(q-\lambda)}{\Gamma(q)} \frac{|G(\lambda, l)|}{|G(0, l+\lambda)|} .
$$

\section{REFERENCES}

1. M. Abramowitz and I. A. Stegun, Handbook of Mathematical Functions, Dover, 1970.

2. H. Bateman, Higher Transcendental Functions, v2, McGraw-Hill, 1953. MR 84h:33001b

3. E. Butkov, Mathematical Physics, Addison-Wesley Publishing Company, 1968.

4. D. Gottlieb and S. Orszag, Numerical Analysis of Spectral Methods: Theory and Applications, SIAM-CBMS, Philadelphia, 1977. MR 58:24983

5. D. Gottlieb, C.-W. Shu, A. Solomonoff and H. Vandeven, On The Gibbs Phenomenon I: recovering exponential accuracy from the Fourier partial sum of a non-periodic analytic function, J. Comput. Appl. Math. 43 (1992), 81-92. MR 94h:42006

6. D. Gottlieb and C.-W. Shu, On The Gibbs Phenomenon III: recovering exponential accuracy in a sub-interval from the spectral partial sum of a piecewise analytic function, SIAM J. Numer. Anal., 33:1 (1996), 280-290. CMP 96:09

7. D. Gottlieb and C.-W. Shu, On The Gibbs Phenomenon IV: recovering exponential accuracy in a sub-interval from a Gegenbauer partial sum of a piecewise analytic function, Math. Comp. 64:211 (1995), 1081-1095. CMP 95:11

8. I. Gradshteyn and I. Ryzhik, Tables of Integrals, Series, and Products, Academic Press, 1980. MR 81g:33001

9. F. John, Partial Differential Equations, Springer-Verlag, 1982. MR 87g:35002 
10. S. Orszag, Fourier Series on Spheres, Mon. Wea. Rev. 102 (1978), 56-75.

11. P. N. Swarztrauber, On the Spectral Approximation of Discrete Scalar and Vector Functions on a Sphere, Siam J. Numer. Anal., 16:6 (1979), 934-949. MR 81c:65011

12. H. Weyl, Die Gibbssche Erscheinung in der Theorie der Kugelfunktionen, Gesammelte Abhandlungen, Springer-Verlag, 1968, 305-320.

Division of Applied Mathematics, Brown University, Providence, Rhode Island 02912

E-mail address: ag@cfm.brown.edu 